\title{
Multiple-Scale Pattern Recognition Applied to Faint Intergranular G-band Structures
}

\author{
B. Bovelet · E. Wiehr
}

Received: 19 December 2006 / Accepted: 2 April 2007 / Published online: 24 August 2007

(C) Springer Science+Business Media B.V. 2007

\begin{abstract}
Small-scale solar magnetic flux concentrations are studied in two-dimensional G-band images at very high spatial resolution and compared with $\mathrm{Ca}$ II $\mathrm{H}$ enhancements. Among 970 small-sized G-band intergranular structures (IgS), 45\% are co-spatial with isolated locations of $\mathrm{Ca}$ II $\mathrm{H}$ excess and thus considered as magnetic (MIgS); they may be even twice as frequent as the known G-band bright points. The IgS are recognized in the G-band image by a new algorithm operating in four steps: (1) A set of equidistant detection levels yields a pattern of primary "cells"; (2) for each cell, the intrinsic intensity profile is normalized to its brightest pixel; (3) the cell sizes are shrunk by a unitary single-intensity clip; (4) features in contact at an appropriate reference level are merged by removal of the respective common dividing lines. Optionally, adjoining structures may be excluded from this merging process (e.g., chains of segmented $\operatorname{IgS}$ ), referring to the parameterized number and intensity of those pixels where enveloping feature contours overlap. From the thus recognized IgS pattern, MIgS are then selected by their local Ca II H contrast and their mean G-band-to-continuum brightness ratio.
\end{abstract}

Keywords Sun: pattern recognition · Intergranular magnetic features

\section{Introduction}

The majority of solar magnetic flux concentrations appear at scales near the spatial resolution limit hitherto achieved. In 403-nm G-band images, magnetic flux concentrations are readily detected as brightenings, mostly located in intergranular lanes (e.g., Langhans, Schmidt, and Tritschler, 2002). Among the magnetic intergranular structures (MIgS) G-band bright points may, however, represent only a subsample ( $c f$. Okunev et al., 2005) since MIgS, which are actually small, will be broadened by the finite spatial resolution, thus appearing G-band faint. Because of the small Zeeman polarization, they are hardly detected in magnetic maps and need to be analyzed in the G-band image. For a sufficient statistical significance, a pattern recognition method is required that detects intergranular structures $(\operatorname{IgS})$

B. Bovelet $\cdot$ E. Wiehr $(\bowtie)$

Institut für Astrophysik, Friedrich-Hund-Platz 1, 37077 Göttingen, Germany

e-mail: ewiehr@astro.physik.uni-goettingen.de 
at intensities even below that of granules. Such a rigid segmentation, however, inevitably subdivides granules into fragments that are hardly distinguishable from the small IgS under study.

A review of existing recognition algorithms has been presented by Berrilli et al. (2005); a new method for the segmentation of photospheric and chromospheric features (in EIT images) has been added by Barra et al. (2005). However, recognized features often show two deficiencies in describing a realistic pattern of solar structures: (i) a clustering of closely neighboring granules and (ii) too wide intergranular lanes. Bovelet and Wiehr (2001; hereafter referred to as Paper I) demonstrated that (i) and (ii) are tightly related: It is the finite spatial resolution achieved in solar images that makes small intergranular lanes appear less intensity-depressed; at increasing spatial resolution, however, small lanes appear as dark as broader ones. Adjacent granules, separated by such pale intergranular lanes, are hardly segmented by any plain pattern recognition. Adapting the algorithm to avoid "artificial conglomerates" of adjacent granules (i) requires the segregation process to stop at an intensity level well above that of pale intergranular lanes; as a consequence, the obtained pattern contains bright features that are smaller than realistic granules and intergranular lanes that are much too broad (ii). In turn, continuing the detection towards lower intensities in order to obtain realistic widths of intergranular lanes, yields an artificial merging (i) of closely neighboring granules.

In Paper I we have demonstrated that multiple level tracking (MLT) avoids such artifacts by tracking any feature, once detected, down to darkest intergranular intensities. Here we present a multiple-scale generalization of MLT for the segmentation of various intensity features from brightest local maxima to faintest structures embedded in the (deep) intergranular lanes.

\section{Basic Idea of the Method}

Automated pattern recognition of even faintest features requires a set of close detection thresholds down to lowest intergranular intensities. Such a procedure, however, inevitably interprets local depressions of larger features as divisions between "substructures." However, if the lowest detection level is chosen sufficiently high (to ensure intergranular lanes of reasonable widths), features with intensities below that minimum threshold would be ignored. The new pattern recognition procedure, MLT_4, overcomes this dilemma in four phases:

(1) Segmentation of features at equidistant levels from maximum to zero intensity; this yields a pattern of cells surrounding each local intensity maximum, finally also covering the intergranular lanes (Figure 1(b)).

(2) Normalization of the pixel intensities of each cell to their maximum; this ensures the following recognition phases to be independent of the individual cellular intensity profiles (Figure 1(c)).

(3) Shrinking of these normalized cells to reasonable sizes by applying a single cutoff threshold to their normalized intensity profiles; this yields feature sizes in accordance with their photometric appearance in the original image (Figure 1(d)). 


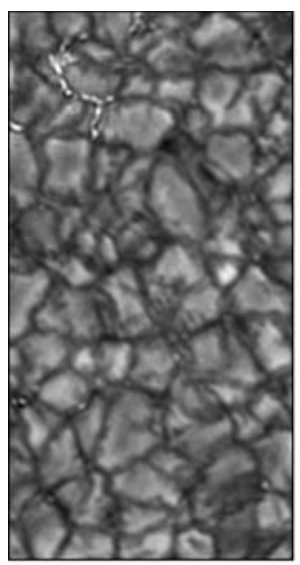

(a)

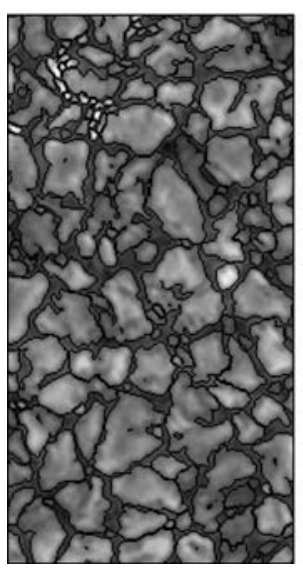

(e)

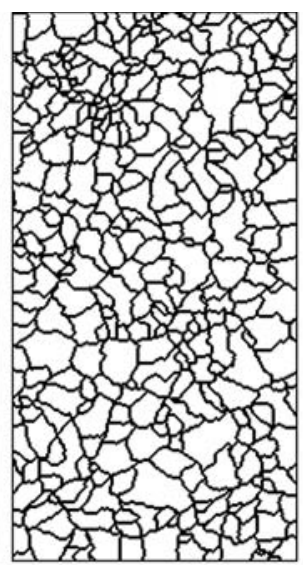

(b)

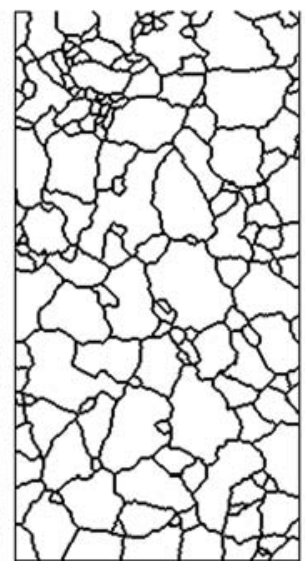

(f)

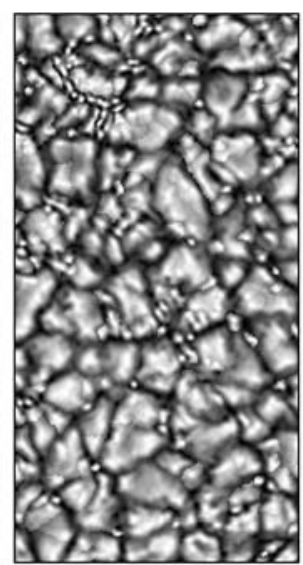

(c)

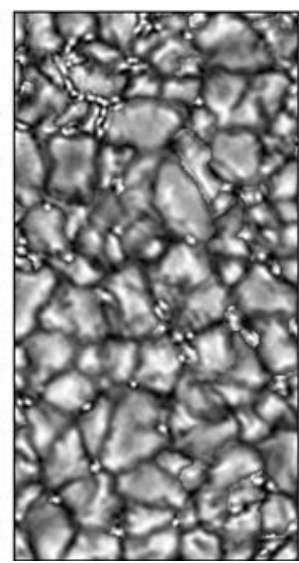

(g)

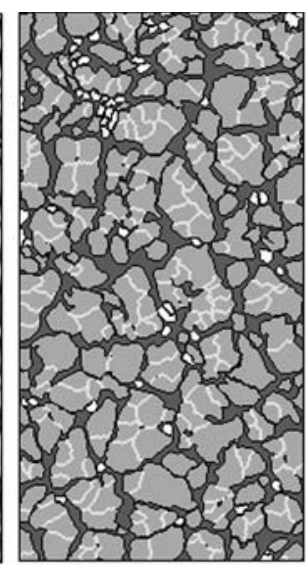

(d)

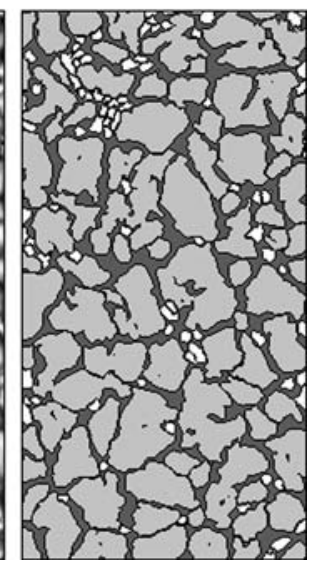

(h)

Figure 1 Demonstration of subsequent phases of the MLT_4 procedure for a reconstructed disk center G-band image (19 October 2005; DOT). (a) $24.1^{\prime \prime} \times 12.6^{\prime \prime}$ subfield; (b) phase 1: pattern of segmented "cells" as detected by multiple level pattern recognition using 20 intensity thresholds; (c) phase 2: intensity normalization for each segmented cell; (d) phase 3: shrunk features (gray) after unitary cut, with features excluded from merging marked white; (e) phase 4: final pattern (black contour pixels), with the division lines (light gray) now removed; (f) expansion of the final features to a cellular pattern; (g) intensity normalization for these cells; (h) selection of particular features, here small intergranular structures (white).

(4) Merging of oversegmented substructures (resulting from the rigid phase 1) whose enveloping contour pixels overlap (white pixel lines in Figure 1(d)); optionally, adjacent structures may be excluded from this merging process (e.g., chains of distinct G-band bright points), referring to the parameterized number and intensity of such joint contour pixels.

In phase 1, equidistant levels are given by an intensity step width used for the photometric top-down detection via MLT. From experience, a set of 15 to 25 levels proves to be sufficient for detecting all features of interest in a map of cells (Figure 1(b)). Since the idea of a "realistic" granular pattern is to some extent subjective, the detection levels may be tuned 
Figure 2 (a) Intrinsic cellular intensity rms as a function of increasing intensity cutoff which gradually shrinks each cell (down to zero at cut rate 1.0$)$; dots represent the respective $\mathrm{rms}$ data per cutoff for the cells segmented in the complete $78.7^{\prime \prime} \times 57.4^{\prime \prime}$ DOT image; the short horizontal gray bar indicates the $1 \sigma$ range at the inflection point of the averaged curve (black line); its second derivative is the lower curved dashed line; the vertical gray bars give the cutoff range applied in phase 3 of MLT_4. (b) Relation between the cutoff and the area contribution of intergranular lanes in the total DOT image; the preferred range yields $70 \%$ granular area (right ordinate).

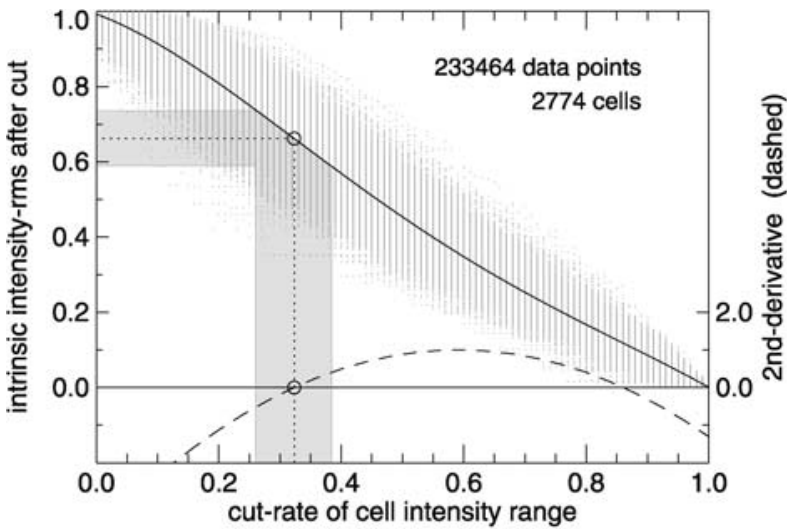

(a)

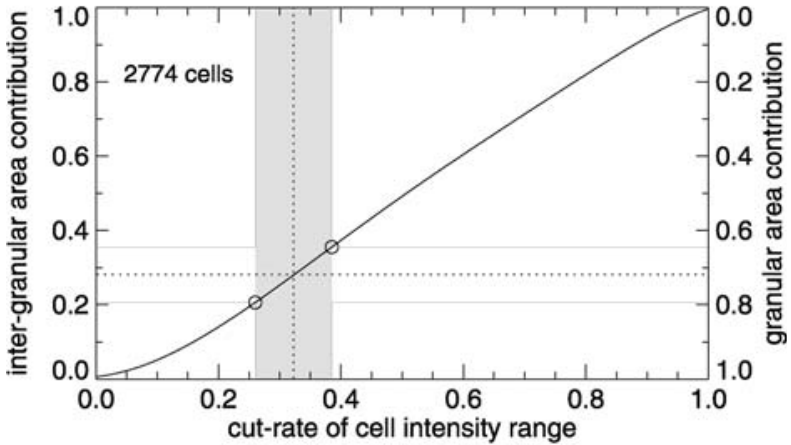

(b)

by means of interactive MLT_4 tools, for example, to reduce irreversible subsegmentations (arising from local enhancements within extended structures).

The shrinking procedure in phase 3 is applied to the intensity pattern from phase 1 after normalization in phase 2 (Figure 1(c)) and is thus independent of the individual brightness profile of each structure. This allows a single cutoff at a reasonable intensity level to be equally applied to all cells, yielding the features in Figure 1(d). For solar granulation, the appropriate cutoff range between 0.26 and 0.38 is derived from a curve that gives the decreasing intensity deviation from the current mean of each cell, when successively shrinking the latter at ascending cutoff levels (Figure 2(a)). The inflection point of this curve corresponds to that threshold which meets the steepest part of the features' photometric profile. Slight variation of the cutoff near that inflection point (gray range in Figure 2(a)) does not significantly vary the extension of the brightness normalized structures; this invariance proves the reliability of the shrinking criterion. A cutoff near the inflection point also reproduces adequate intergranular lanes and thus a realistic granular area fraction (Figure 2(b)).

The merging in phase 4 is applied to adjacent substructures whose enveloping contour pixels overlap (white pixel lines in Figure 1(d)) even after the shrinking process in phase 3. This procedure readily meets the risk of also merging those adjacent features that are reasonably considered as individual solar structures. Hence, appropriate criteria are introduced to exclude particular structures from the merging process, for example, chains of distinct BP (cf. Bovelet and Wiehr, 2003; hereafter referred to as Paper II). 


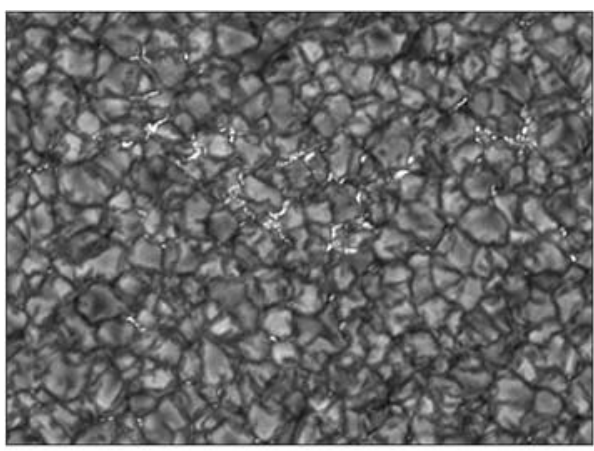

(a)

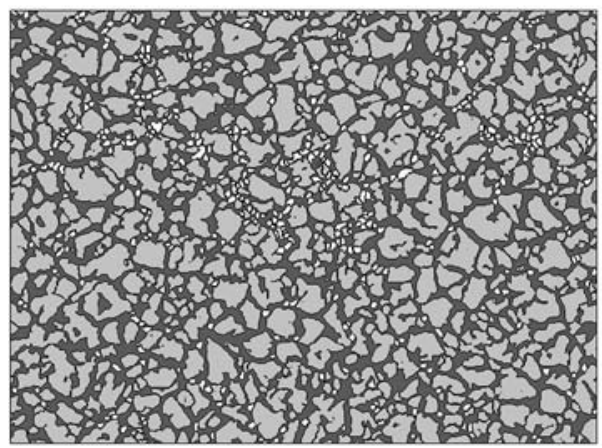

(c)

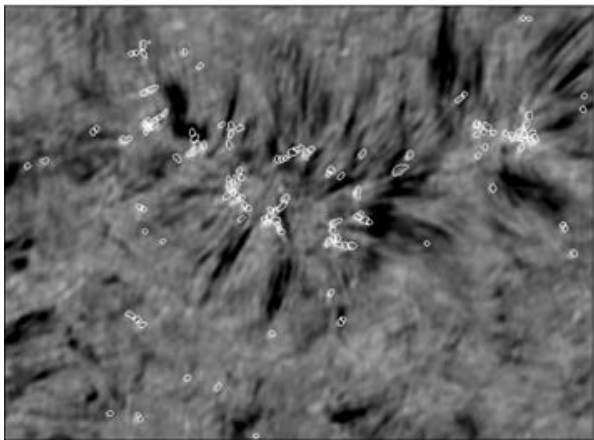

(e)

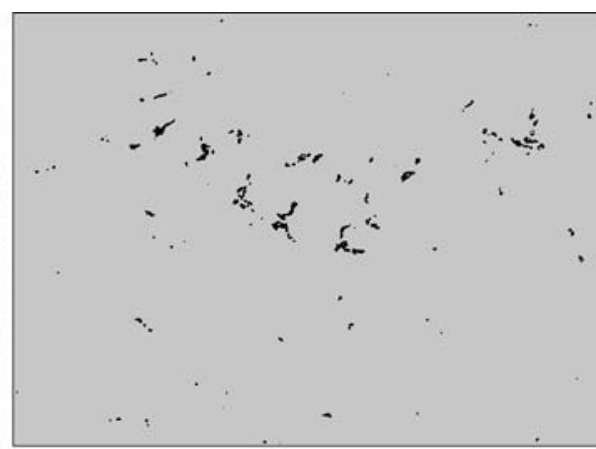

(b)

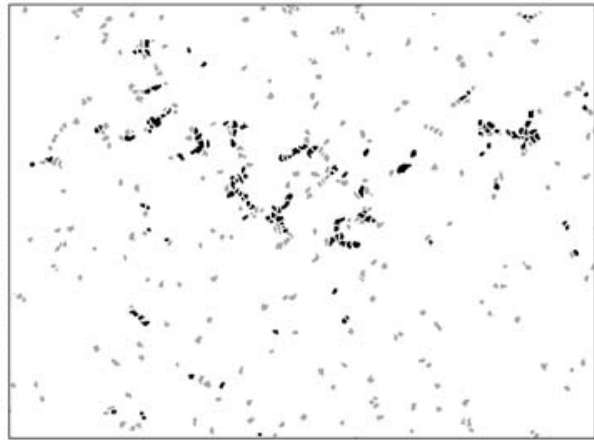

(d)

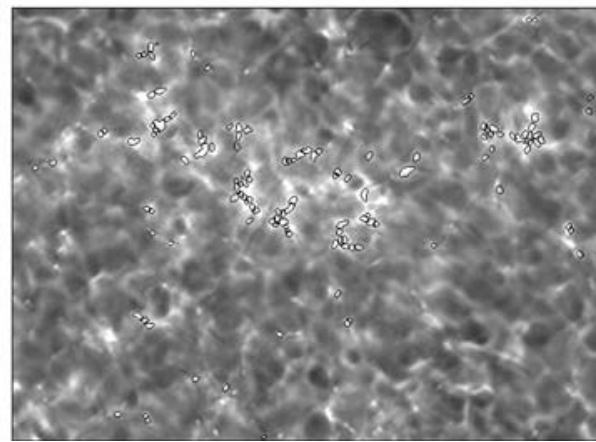

(f)

Figure 3 Application of the selection procedure to intergranular structures $(\mathrm{IgS})$ in a $43.2^{\prime \prime} \times 31.8^{\prime \prime}$ subfield (a) of the original G-band image with pronounced activity. (b) Difference map "blue-continuum minus G-band." (c) Selected IgS (white) within the granular pattern. (d) Subsamples of bright (black) and faint (gray) IgS. Simultaneously observed (e) $\mathrm{H} \alpha-0.7 \AA$ and (f) $\mathrm{Ca}$ II H images, with G-band bright IgS outer contoured (black).

\section{Application of MLT_ 4 to Intergranular Structures}

\section{1. $\operatorname{IgS}$ in an Active Subregion}

We apply the MLT_4 algorithm to an active subregion (Figure 3(a)) of the full G-band image; the difference map "blue-continuum minus G-band" reveals pronounced magnetic fea- 
tures (black in Figure 3(b)). The MLT_4 pattern covers solar features independent of their brightness and size or whether they are granular and intergranular, (Figure 3(c)). Among these, we select small intergranular structures (IgS, white in Figure 3(c)), using their strong "local contrast" ( $c f$. Paper II), and weighting them by their reciprocal size (1/px) to exclude extended features (e.g., bright granular fragments).

To distinguish G-band bright from G-band faint IgS (Figure 3(d)), we apply an intensity threshold at 1.17 [I_phot], being adapted to an optimal representation of such structures that are visible in the difference map (Figure 3(b)) (i.e., features known as BP). Superposing the pattern of these G-band bright IgS with the simultaneously observed Ca II H image (Figure 3(f)) proves their (known) location at $\mathrm{Ca}$ II $\mathrm{H}$ enhancements. In the $\mathrm{H} \alpha-0.7 \AA$ image (Figure 3(e)) only a minority of the G-band bright IgS coincide with enhancements.

\subsection{IgS in the Full G-band Image}

To check the relation of IgS with locations of a Ca II H and an H $\alpha-0.7 \AA$ excess at a statistically high significance, we apply this procedure to the full DOT image $\left(78.7^{\prime \prime} \times 57.4^{\prime \prime}\right)$. The recognized 718 G-band faint structures among the sample of $970 \mathrm{IgS}$ from this image are compared with the simultaneously observed $\mathrm{Ca}$ II $\mathrm{H}$ image. Assuming a $\mathrm{Ca}$ II $\mathrm{H}$ excess as the chromospheric signature of magnetic flux concentrations (e.g., Knölker, Schüssler, and Weisshaar, 1988), we now select those IgS that appear bright in Ca II H. Since the region is located at disk center, spatial displacements with height cannot interfere.

The Ca II H image shows numerous enhancements, among which many are known to be the signature of nonmagnetic chromospheric heating (e.g., of acoustic nature). We thus restrict the selection of magnetic structures to those $\mathrm{Ca}$ II $\mathrm{H}$ enhancements that are isolated and of small size. However, we cannot exclude that the chromospheric brightening of some intergranular magnetic flux concentrations may be superposed by those extended brightenings of nonmagnetic origin, yet excluded by our criterion. As a consequence, our selection of magnetic intergranular structures, MIgS, represents a lower limit ( $c f$. Table 1).

Among the entire sample of IgS (G-band faint and bright) at least $45 \%$ are MIgS. The remaining IgS, assumed to be of nonmagnetic nature, either exhibit no Ca II $\mathrm{H}$ enhancement or are embedded in a region of spatially extended, nonlocal Ca II H excess ( $c f$. Table 1). A scatter plot of the G-band brightness versus the Ca II H excess (Figure 4(a)) shows for the MIgS (black dots) a largely linear relation, indicating that increasing G-band intensity corresponds to higher chromospheric brightening.

An alternative distinction between magnetic and nonmagnetic $\operatorname{IgS}$, resulting from theoretical modeling, is based on the ratio of the G-band and the neighboring continuum intensity. In the corresponding scatter plot, two branches represent the strong magnetic flux concentrations and the weakly magnetized atmosphere, and these intersect for low intensities (cf. Shelyag et al., 2004). Applying this criterion to the observed $970 \operatorname{IgS}$ (Figure 4(b)), we find that about $10 \%$ of the IgS appear on the, respectively, opposite branch: A definite distinction would require a priori knowledge of their magnetic identity. However, even when artificially separating the two branches at a reasonable ratio (e.g., 1.1), the total number of MIgS remains almost unchanged.

Applying this ratio as a magnetic criterion to the sample of $970 \mathrm{IgS}$ gives a histogram of their G-band brightness (Figure 5) that shows a largely smooth distribution of $479 \mathrm{MIgS}$, thus evidently forming a unitary entity. The 238 G-band faint features among these MIgS complete the sample of $241 \mathrm{G}$-band bright magnetic structures (the latter being identified as igBP by Wiehr, Bovelet, and Hirzberger, 2004), which then represent half of the MIgS, only. 
Table 1 Scheme of intergranular structures (IgS) recognized by MLT_4 in the G-band image and classified by their spatially corresponding $\mathrm{Ca}$ II $\mathrm{H}$ enhancements; the numbers in italics give the quantity of IgS per subsample.

\begin{tabular}{|c|c|c|c|}
\hline \multicolumn{4}{|c|}{ Inter-granular Structures (IgS) } \\
\hline \multicolumn{3}{|c|}{ G-band faint } & G-band bright ('BP') \\
\hline \multicolumn{3}{|c|}{$-718-$} & \multirow{5}{*}{ Ca II $\mathrm{H}$ bright } \\
\hline \multirow{4}{*}{$\begin{array}{l}\text { Ca II H faint } \\
-213-\end{array}$} & \multirow{2}{*}{\multicolumn{2}{|c|}{$\begin{array}{c}\text { Ca II H bright } \\
-505-\end{array}$}} & \\
\hline & & & \\
\hline & non-isolated & isolated & \\
\hline & $-324-$ & $-181-$ & \\
\hline \multicolumn{2}{|c|}{ non-magnetic IgS } & \multicolumn{2}{|c|}{ Magnetic IgS (MIgS) } \\
\hline \multicolumn{2}{|c|}{$-537-$} & \multicolumn{2}{|r|}{$-433-$} \\
\hline
\end{tabular}

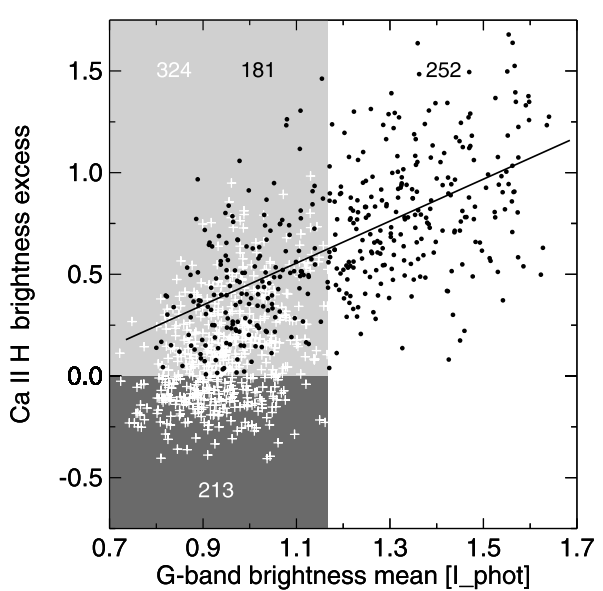

(a)

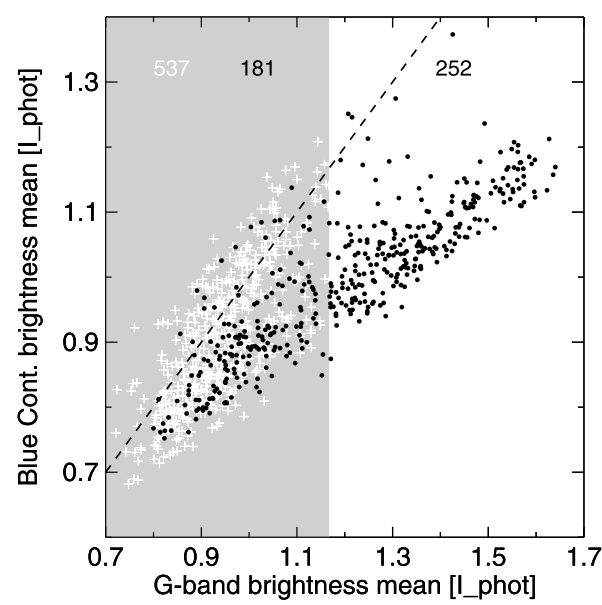

(b)

Figure 4 Scatter-plots of the G-band brightness (mean per feature) versus the Ca II H (a) and versus the blue continuum excess (b) of intergranular structures $(\mathrm{IgS})$ in the total 78.7" $\times 57.4^{\prime \prime}$ image: G-band bright $\mathrm{IgS}$ (BP) are distinguished from G-band faint ones (darker gray grounded); among the latter, nonmagnetic (white crosses) are selected by missing or nonisolated Ca II H excess; the magnetic IgS (MIgS) reveal a linear correlation of the G-band brightness with the Ca II H excess (black line). The continuum brightness of the such classified IgS (b) allows us to assign them to two MHD modeled branches; the nonmagnetic IgS scatters around the 1.0 ratio (dashed line).

By considering the H $\alpha-0.7 \AA$ brightness of the recognized IgS, an analog scatter plot of the G-band brightness versus the corresponding H $\alpha-0.7 \AA$ excess exhibits no significant correlation: At most $50 \%$ of the G-band bright $\operatorname{IgS}$ (i.e., BP) also occur bright in H $\alpha$ - 
Figure 5 Number distribution of the mean G-band brightness of $\mathrm{IgS}$, here distinguished just by their G-band excess over the continuum brightness: ratio $<1.1$ (upper panel) representing 491 nonmagnetic $\mathrm{IgS}$, and ratio $\geq 1.1$ giving $479 \mathrm{MIgS}$ (lower panel), among which 241 are G-band bright (known as BP; lower right). The data points represent counts per bin size of 80 maximum-scaled histograms with different bin sizes, which are overplotted to provide sufficient invariance from subjective sampling.

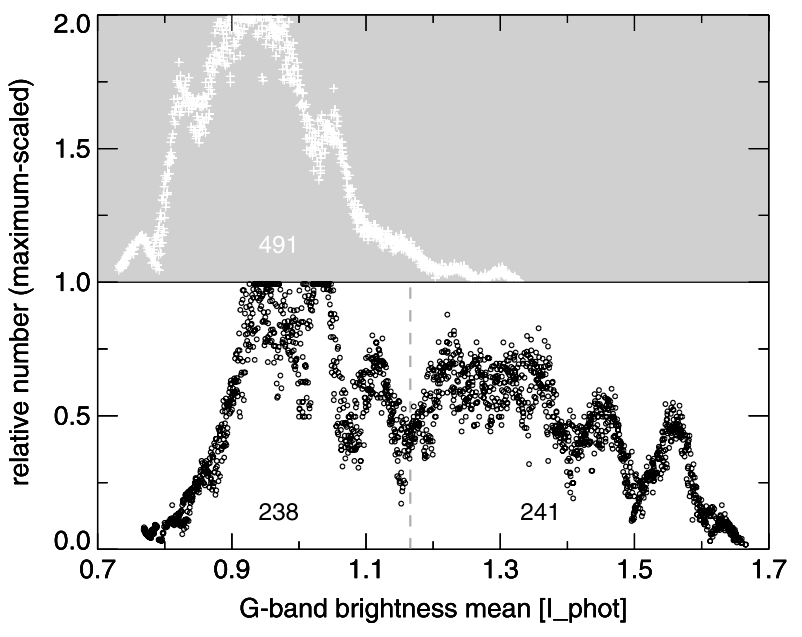

$0.7 \AA$. Hence, the majority of MIgS is not detectable in the H $\alpha-0.7 \AA$ image, thus suggesting that the H $\alpha-0.7 \AA$ brightness is not a suitable indicator for magnetic flux concentrations.

\section{Conclusions}

The four-phase multiple-scale pattern recognition of MLT_4 detects numerous small-scale intergranular structures. By taking the ratio of the G-band and the neighboring continuum brightness as a criterion for magnetic structures, the majority of $\operatorname{IgS}$ with ratio $\geq 1.1$ appear locally bright in CaII H. This is nicely seen in Figure 6, showing another (barely active) subregion of the DOT image with numerous magnetic IgS. In turn, $\operatorname{IgS}$ with ratio $<1.1$ (white contoured in Figure 6(d)) mostly lack a Ca II H excess; the few exceptions may either be of acoustic nature or correspond to the magnetic branch (ratio $\geq 1.1$ ). The entity of these magnetic intergranular structures (G-band bright and G-band faint MIgS) amounts to almost twice the number of features hitherto known as BP, the latter thus significantly undersampling the occurrence of small-scale magnetic flux concentrations.

The H $\alpha-0.7 \AA$ brightness undersamples the MIgS even more significantly. For active areas, it is known that the high-reaching arches of dark mottles may hide underlying features. This, however, does not hold for the G-band faint MIgS, which are located well outside pronounced activity. Hence, neither the H $\alpha-0.7 \AA$ nor the G-band intensity excess provides a criterion that is sufficient for the identification of small-scale magnetic flux concentrations. MIgS that are faint in the G-band image may appear brighter and smaller at higher spatial resolution (see, e.g., Puschmann and Wiehr, 2006), thus preserving their integrated intensity flux. A powerful method of photometric pattern recognition, such as MLT_4, ensures a sufficient statistical significance even at higher spatial resolutions as achieved, for example, in the HINODE images and expected from larger solar telescopes.

The main procedure MLT_4 and the implemented function MLT_4F may be downloaded from http://www.gwdg.de/ bbovele, using the login name user and password mlt4. The codes are designed to run under PV-WAVE 6.0 (by Visual Numerics Inc.) or IDL 2.0 (by Research Systems Inc.) and higher releases. 


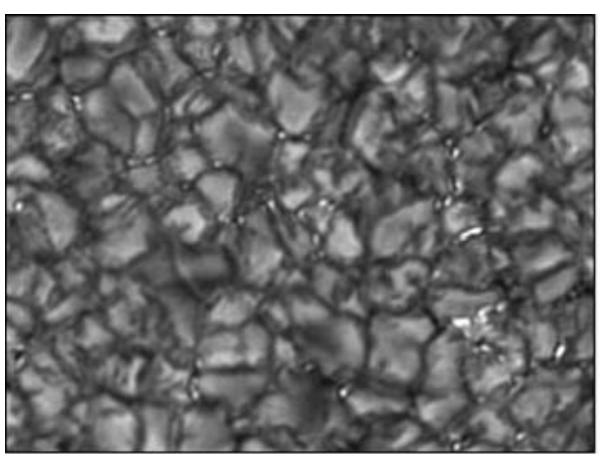

(a)

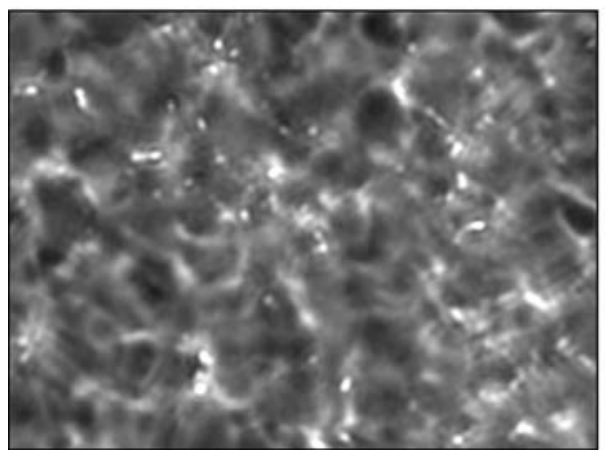

(c)

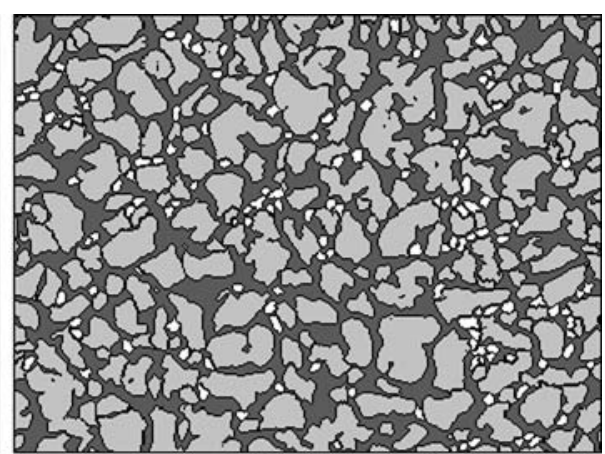

(b)

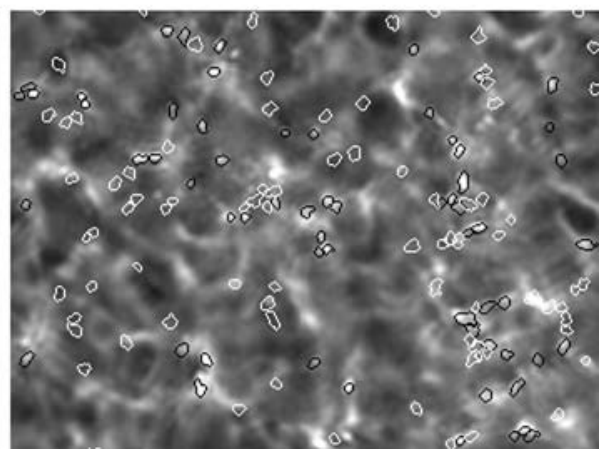

(d)

Figure 6 (a) Subfield $\left(24.9^{\prime \prime} \times 18.5^{\prime \prime}\right)$ of the G-band image; (b) pattern recognized by MLT 4 with intergranular structures (IgS, white) selected by their size-weighted local G-band contrast; (c) corresponding subfield in the Ca II H image; (d): superimposed outer contours of magnetic IgS, distinguished by their ratio of G-band to continuum brightness: ratio $<1.1$ (white contours) and ratio $\geq 1.1$ (black contours).

Acknowledgements We are greatly indebted to Dr. Peter Sütterlin (Utrecht) who kindly put a brilliant set of aligned speckle-reconstructed images (DOT) at our disposal.

\section{References}

Barra, V., Delouille, V., Hochedez, J.-F., Chainais, P.: 2005. In: Danesy, D., Poedts, S., De Groof, A., Andries, J. (eds.) Proceedings of the 11th European Solar Physics Meeting 'The Dynamic Sun: Challenges for Theory and Observations,' Leuven, Belgium, 11-16 September 2005, ESA SP-600, ESA, Noordwijk, 77.1, CDROM.

Berrilli, F., Del Moro, D., Florio, A., Santillo, L.: 2005, Solar Phys. 228, 81

Bovelet, B., Wiehr, E.: 2001, Solar Phys. 201, 13 (Paper I).

Bovelet, B., Wiehr, E.: 2003, Astron. Astrophys. 412, 249 (Paper II).

Knölker, M., Schüssler, M., Weisshaar, E.: 1988, Astron. Astrophys. 194, 256.

Langhans, K., Schmidt, W., Tritschler, A.: 2002, Astron. Astrophys. 394, 1069.

Okunev, O.V., Domínguez Cerdeña, I., Puschmann, K.G., Kneer, F., Sánchez Alemeida, J.: 2005, Astron Nachr. 326, 205.

Puschmann, K.G., Wiehr, E.: 2006, Astron. Astrophys. 445, 337.

Shelyag, S., Schüssler, M., Solanki, S.K., Berdyugina, S.V., Vögler, A.: 2004, Astron. Astrophys. 427, 335.

Wiehr, E., Bovelet, B., Hirzberger, J.: 2004, Astron. Astrophys. 422, 63. 